\title{
A RESTRICTION ON THE SCHUR MULTIPLIER OF NILPOTENT LIE ALGEBRAS*
}

\author{
PEYMAN NIROOMAND ${ }^{\dagger}$ AND FRANCESCO G. RUSSO ${ }^{\ddagger}$
}

\begin{abstract}
An improvement of a bound of Yankosky (2003) is presented in this paper, thanks to a restriction which has been recently obtained by the authors on the Schur multiplier $M(L)$ of a finite dimensional nilpotent Lie algebra $L$. It is also described the structure of all nilpotent Lie algebras such that the bound is attained. An important role is played by the presence of a derived subalgebra of maximal dimension. This allows precision on the size of $M(L)$. Among other results, applications to the non-abelian tensor square $L \otimes L$ are illustrated.
\end{abstract}

Key words. Schur multiplier, Nilpotent Lie algebras, Derived subalgebra, Non-abelian tensor product.

AMS subject classifications. 17B30, 17B60, 17B99

1. Introduction and terminology. The classification of a finite dimensional Lie algebra $L$ via its Schur multiplier $M(L)$ is a new field of interest in literature. On one hand, several authors investigated the deep connections among $L$ and the structure of a finite $p$-group $G$ ( $p$ a prime) with respect to its Schur multiplier $M(G)$. For instance, [7, Chapters 3 and 4] is a classic reference, which describes the aspects of the commutator calculus on Lie algebras and the influence on the classification of finite $p$-groups. On the other hand, a direction of research, in a certain sense opposite, was introduced by Batten et al., who illustrated in [1, Theorem 5] that a bound for $M(G)$ can be translated in terms of a bound for the $\operatorname{dimension} \operatorname{dim}(L)=n \geq 1$ of $L$.

Assigned $t(L)=\frac{1}{2} n(n-1)-\operatorname{dim}(M(L)),[1$, Theorem 3] shows that $t(L)=1$ if and only if $L \cong H(1)$, where $H(1)$ is the Heisenberg algebra of dimension 3 . We recall that $L$ is called Heisenberg provided that $L^{2}=Z(L)$ and $\operatorname{dim}\left(L^{2}\right)=1$. Such algebras are odd dimensional with basis $v_{1}, \ldots, v_{2 m}, v$ and the only non-zero multiplication between basis elements is $\left[v_{2 i-1}, v_{2 i}\right]=-\left[v_{2 i}, v_{2 i-1}\right]=v$ for $i=1,2, \ldots, m$. The symbol $H(m)$ denotes the Heisenberg algebra of dimension $2 m+1$. Moreover, $[1$, Theorem 5] shows that $t(L)=2$ if and only if $L \cong H(1) \oplus A(1)$, where $A(1)$ is an

${ }^{*}$ Received by the editors on August 7, 2010. Accepted for publication on December 14, 2010. Handling Editor: Robert Guralnick.

†School of Mathematics and Computer Science, Damghan University, Damghan, Iran (niroomand@du.ac.ir, p_niroomand@yahoo.com).

${ }^{\ddagger}$ Department of Mathematics, University of Palermo, via Archirafi 14, 90123, Palermo, Italy (francescog.russo@yahoo.com). 
abelian algebra of $\operatorname{dim}(A(1))=1$. More generally, we denote with the symbol $A(n)$ an abelian algebra of $\operatorname{dim}(A(n))=n$.

$[1$, Theorem 3] was subject to a series of successive generalizations in $[1,2,5,8$, $13,18]$, which are devoted to detect the structure of $L$, when values of $t(L)$ higher than 2 are considered. On another hand, [1, 2, 5, 8, 13, 18] deal with bounds on $\operatorname{dim}(M(L))$ and sometimes it is possible to adapt these bounds in order to study other important constructions of topology and $K$-theory, investigated in $[4,11,12,16]$, where restrictions on the second homology group are involved.

The main strategies in $[1,2,5,8,18]$ are summarized in $[1$, Section 1$]$, where it is illustrated that the origin of the investigations goes back to the $p$-groups classified by Zhou in [19]. A similar parallel is possible, when we compare [9, Main Theorem] with [13, Theorem 3.1]. Therefore, we get to the subject of the present paper: we improve [13, Theorem 3.1], overlapping a situation for $p$-groups with large derived subgroup, recently investigated in [10]. We will see that significant restrictions on $\operatorname{dim}(M(L))$ can be obtained, in presence of a derived subalgebra of maximal dimension and will note that Yankosky described a special case of this situation almost seven years ago. We will show that it is possible to obtain his bound [18, Corollary 3.7] in a wider perspective and in a short way, when we involve different techniques. These are our main contributions, which are placed in Section 3, after we recall some fundamental tools in Section 2. Finally, an application is shown in Section 4, where the constructions in $[4,11,12,16]$ are analyzed in virtue of the results in Section 3.

2. Preliminaries. We list the classifications, which are necessary to our aims. In the case of groups, we have the following result.

Proposition 2.1 (See [9], Main Theorem). A p-group G of order $p^{n}$ with the abelianization $G^{a b}=G / G^{\prime}$ of order $p^{d}$ has $|M(G)| \leq p^{\frac{1}{2}(2 n-d-2)(d-1)+1}$, where $d \leq n-1$. In particular, $|M(G)| \leq p^{\frac{1}{2}(n-1)(n-2)+1}$ and the equality holds in this last bound if and only if $G=H \times Z$, where $H$ is extra special of order $p^{3}$ and exponent $p$ and $Z$ is an elementary abelian $p-$ group.

In the case of Lie algebras, we proceeded by analogy and found the following classification with different techniques and arguments.

TheOREM 2.2 (See [13], Theorem 3.1). For an n-dimensional non-abelian nilpotent Lie algebra $L$ such that $\operatorname{dim}\left(L / L^{2}\right)=d$, we have

$$
\operatorname{dim}(M(L)) \leq \frac{1}{2}(2 n-d-2)(d-1)+1 .
$$

Moreover, if $d=n-1$, then the equality holds if and only if $L \cong H(1) \oplus A(n-3)$.

The next contribution, recently obtained in [10], will allow us to shed light on a 
special situation of Theorem 2.2, related to [18, Corollary 3.7].

Proposition 2.3 (See [10], Theorem 2.1). Assume that a $p$-group $G$ of order $|G|=p^{n}(n \geq 4)$ has $\left|G^{\prime}\right|=p^{n-2}$. Then $|M(G)| \leq p^{n-2}$, if $p \neq 2$, and $|M(G)| \leq$ $p^{n-3}$, if $p=2$.

For the special case of filiform Lie algebras and $p$-groups of maximal class, similar results have been shown by Bosko in [3], where a special case has been treated. In fact, the reader may compare Proposition 2.3 and Theorem 3.1 below with [3, Theorems 10 and 12]. Now some general considerations are recalled from [13].

REMARK 2.4. The Schur multiplier of the direct product of two finite groups is equal to the direct product of the Schur multipliers of the two factors plus the tensor product of the abelianization of the two groups (see [6, Theorem 2.2.10]). This fact comes from basic homology and is known as the Künneth Formula (see [15]): it is true also for two finite dimensional Lie algebras $H$ and $K$. The Künneth Formula was originally obtained by Schur in 1904 (see [17]). We recall that $\otimes_{\mathbb{Z}}$ denotes the usual tensor product of abelian Lie algebras and $\oplus$ their direct sum. Then we have

$$
M(H \oplus K)=M(H) \oplus M(K) \oplus\left(H / H^{2} \otimes_{\mathbb{Z}} K / K^{2}\right) .
$$

In particular we may deduce the following two facts.

Proposition 2.5 (See [13], Theorem 2.2). Let $A$ and $B$ be two finite dimensional Lie algebras. Then

$$
\operatorname{dim}(M(A \oplus B))=\operatorname{dim}(M(A))+\operatorname{dim}(M(B))+\operatorname{dim}\left(A / A^{2} \otimes_{\mathbb{Z}} B / B^{2}\right) .
$$

Corollary 2.6 (See [13], Corollary 2.3). Let L be a finite dimensional Lie algebra, $K$ be a central ideal of $L$ and $H=L / K$. Then

$$
\operatorname{dim}(M(L))+\operatorname{dim}\left(L^{2} \cap K\right) \leq \operatorname{dim}(M(H))+\operatorname{dim}(M(K))+\operatorname{dim}\left(H / H^{2} \otimes_{\mathbb{Z}} K\right) .
$$

3. Main theorems. The following result was motivated by the presence of a derived subalgebra of maximal dimension and originated from the situation in Proposition 2.3 .

TheOREM 3.1. An $n$-dimensional nilpotent Lie algebra $L$ with $\operatorname{dim}\left(L^{2}\right)=n-2$ and $n \geq 4$ has $\operatorname{dim}(M(L)) \leq \operatorname{dim}\left(L^{2}\right)$.

Proof. We perform induction on $n$. Assume $n=4$. Then Theorem 2.2 allows us to conclude that $\operatorname{dim}(M(L)) \leq 3$. From $[5$, Theorem 1] there is no Lie algebra $L$ with 
$\operatorname{dim}\left(L^{2}\right)=2$, when $\operatorname{dim}(M(L))=3$. Therefore, $\operatorname{dim}(M(L)) \leq 2$ and so the result is true for $n=4$.

From Corollary 2.6 we have, for a central ideal of dimension 1 in $L^{2}$,

$$
\begin{gathered}
1+\operatorname{dim}(M(L)) \leq \operatorname{dim}(M(L / K))+\operatorname{dim}(M(K))+\operatorname{dim}\left(\frac{L / K}{(L / K)^{2}} \otimes_{\mathbb{Z}} K\right) \\
\leq \operatorname{dim}\left(L^{2} / K\right)+2,
\end{gathered}
$$

by the induction hypothesis. Hence,

$$
\operatorname{dim}(M(L)) \leq 1+\operatorname{dim}\left(L^{2} / K\right)=\operatorname{dim}\left(L^{2}\right)
$$

and the result follows. $\square$

We note that the above bound improves that of Yankosky, quoted below.

Lemma 3.2 (See [18], Corollaries 3.7, 3.8). An n-dimensional nilpotent Lie algebra $L$ with $\operatorname{dim}\left(L^{2}\right)=n-2$ has $\operatorname{dim}(M(L)) \leq \operatorname{dim}\left(L^{2}\right)+1$. Furthermore, if $\operatorname{dim}\left(L / L^{2}\right)=d$, then

$$
\operatorname{dim}(M(L)) \leq \frac{d-d^{2}+2 d n-2 n}{2}
$$

The inequality in Theorem 2.2 is, in general, better than the inequality in Lemma 3.2 , unless that $L \cong H(1)$. This case is described with details by the next result.

Lemma 3.3. The bound in Theorem 2.2 is less than the bound in Lemma 3.2 unless $d=2$.

Proof. We have

$$
\frac{1}{2}(2 n-d-2)(d-1)+1<\frac{1}{2}\left(d-d^{2}+2 n d-2 n\right)
$$

and short calculations show that the quantity which appears in the left side of the above inequality is less than the quantity which appears in the right side if and only if $d>2$. The result follows.

Corollary 3.4. Let $L$ be an $n$-dimensional nilpotent Lie algebra. Then the bound in Theorem 2.2 is less than the bound in Lemma 3.2 for all $n \geq 4$. For $n=3$ the bounds are equal and $L \simeq H(1)$.

Proof. It is obvious that the bounds are equal for $n=3$. In this case $L \simeq H(1)$. Thus, we assume $n \geq 4$. Lemma 3.3 shows that the two bounds are equal when $d=2$. 
Thus Theorem 2.2 allows us to conclude that $L \not H(1) \oplus A(n-3)$, since $\operatorname{dim}\left(L^{2}\right) \geq 2$. Still Theorem 2.2 implies $\operatorname{dim}(M(L)) \leq \frac{1}{2}(2 n-d-2)(d-1)$ and this is less than the value in Lemma 3.2. $\square$

The above arguments allow us to have a short proof of Yankosky's Theorem, that is, of Lemma 3.2, and to characterize the structure of all Lie algebras which achieve that bound.

THEOREM 3.5. An $n$-dimensional nilpotent Lie algebra $L$ such that $\operatorname{dim}\left(L / L^{2}\right)=$ $d$ has

$$
\operatorname{dim}(M(L)) \leq \frac{d-d^{2}+2 d n-2 n}{2}
$$

and the equality holds if and only if $L \simeq A(n)$ or $L \simeq H(1)$.

Proof. First assume that $L$ is an abelian Lie algebra. From [1, Lemma 3], we deduce that $L \simeq A(n)$. Hence, we may assume that $L$ is non-abelian. In the case $d>2$ the result follows from Theorem 2.2 and Corollary 3.4. In the case $d=2$ the result follows from Lemma 3.3 .

In Theorems 3.1 and 3.5 we proved some upper bounds for $\operatorname{dim}(M(L))$ by looking only at $\operatorname{dim}\left(L / L^{2}\right)=d$ and $\operatorname{dim}(L)=n$. Now we involve also $\operatorname{dim}(Z(L))=t \geq 1$ and $\operatorname{dim}\left(Z(L) \cap L^{2}\right)=s \geq 1$.

THEOREM 3.6. An $n$-dimensional nilpotent Lie algebra $L$ such that $\operatorname{dim}\left(L / L^{2}\right)=$ $d, \operatorname{dim}(Z(L))=t$ and $\operatorname{dim}\left(Z(L) \cap L^{2}\right)=$ s has

$$
\operatorname{dim}(M(L)) \leq \frac{d-d^{2}+2 d n-2 n}{2}-(n-d)(t-s)
$$

In particular, Theorem 3.5 is obtained for $s=t$.

Proof. First we assume that $L$ is abelian. Hence, $d=n$ and the result follows from [1, Lemma 3]. Then we assume that $L$ is non-abelian. Still [1, Lemma 3] allows us to conclude that the abelian Lie algebra $L / L^{2}$ should satisfy the relation

$$
\operatorname{dim}\left(M\left(L / L^{2}\right)\right)=\frac{1}{2} d(d-1)
$$

By invoking [18, Corollary 3.3],

$$
\operatorname{dim}(M(L)) \leq \operatorname{dim}\left(M\left(L / L^{2}\right)\right)+\operatorname{dim}\left(L^{2}\right)\left(\operatorname{dim}\left(\frac{L}{Z(L)+L^{2}}\right)-1\right)
$$

and then we may proceed as follows

$=\frac{1}{2} d(d-1)+(n-d)(n-(t+n-d-s)-1)=\frac{d-d^{2}+2 d n-2 n}{2}-(n-d)(t-s)$.

The remaining part $s=t$ of the proof is straightforward. $\square$ 
4. Applications to non-abelian tensor squares. In this section, we recall some constructions from $[4,11,12,16]$ and illustrate the consequences of Theorems $3.1,3.5$ and 3.6 in a different context.

Let $F$ be a fixed field, $L, K$ be two Lie algebras (not necessarily of finite dimension), $c \in F, l, l^{\prime} \in L, k, k^{\prime} \in K$ and $[\cdot, \cdot]$ be the usual Lie bracket. By an action of $L$ on $K$, we mean an $F$-bilinear map

$$
(l, k) \in L \times K \mapsto{ }^{l} k \in K
$$

satisfying

$$
\begin{gathered}
{ }^{\left[l, l^{\prime}\right]} k={ }^{l}\left({ }^{l^{\prime}} k\right)-{ }^{l^{\prime}}\left({ }^{l} k\right), \\
{ }^{l}\left[k, k^{\prime}\right]=\left[{ }^{l} k, k^{\prime}\right]+\left[k,{ }^{l} k^{\prime}\right] .
\end{gathered}
$$

Clearly, if $L$ is a subalgebra of some Lie algebra $P$ and $K$ is an ideal in $P$, then the Lie multiplication in $P$ induces an action of $L$ on $K$. In fact, $l$ acts on $k$ by ${ }^{l} k=[l, k]$. Let $L$ and $K$ be Lie algebras acting on each other, and on themselves by Lie multiplications. Then these actions are said to be compatible if ${ }^{k} k^{\prime}=k^{\prime}\left({ }^{l} k\right)$ and ${ }^{l}{ }^{k} l^{\prime}={ }^{l^{\prime}}\left({ }^{k} l\right)$. It is obvious that if $L$ and $K$ are both ideals of some Lie algebra, then the Lie multiplication gives rise to compatible actions. For each Lie algebra $Q$, we call a bilinear function $\varphi: K \times L \rightarrow Q$ a Lie pairing if

$$
\begin{gathered}
\varphi\left(\left[l, l^{\prime}\right], k\right)=\varphi\left(l,{ }^{l^{\prime}} k\right)-\varphi\left(l^{\prime},{ }^{l} k\right), \\
\varphi\left(l,\left[k, k^{\prime}\right]\right)=\varphi\left({ }^{k^{\prime}} l, k\right)-\varphi\left({ }^{k} l, k^{\prime}\right), \\
\varphi\left({ }^{k} l,{ }^{l^{\prime}} k^{\prime}\right)=-\left[\varphi(l, k), \varphi\left(l^{\prime}, k^{\prime}\right)\right]
\end{gathered}
$$

The non-abelian tensor product $L \otimes K$ of $L$ and $K$ is the Lie algebra generated by the symbols $l \otimes k$ with defining relations

$$
\begin{gathered}
c(l \otimes k)=c l \otimes k=l \otimes c k, \\
\left(l+l^{\prime}\right) \otimes k=l \otimes k+l^{\prime} \otimes k, \\
l \otimes\left(k+k^{\prime}\right)=l \otimes k+l \otimes k^{\prime}, \\
{ }^{l} l^{\prime} \otimes k=l \otimes{ }^{l^{\prime}} k-l^{\prime} \otimes{ }^{l} k,
\end{gathered}
$$




$$
\begin{aligned}
& l \otimes{ }^{k} k^{\prime}={ }^{k^{\prime}} l \otimes k-{ }^{k} l \otimes k^{\prime}, \\
& {\left[l \otimes k, l^{\prime} \otimes k^{\prime}\right]=-{ }^{k} l \otimes{ }^{l^{\prime}} k^{\prime} .}
\end{aligned}
$$

In the case $L=K$ and all actions are given by Lie multiplication, $L \otimes L$ is called non-abelian tensor square of $L$. It is opportune to note that we find the usual abelian tensor product $L \otimes_{\mathbb{Z}} K$, when $L$ and $K$ are abelian and the actions are compatible and trivial.

Following the terminology in [4, Section 2], $L \square L=\langle l \otimes l \mid l \in L\rangle$ is a submodule of $L \otimes L$ and lies in the center $Z(L \otimes L)$ of $L \otimes L$. In particular, $L \square L$ is an ideal of $L \otimes L$ and we may consider the Lie algebra quotient

$$
L \wedge L=\frac{L \otimes L}{L \square L}=\left\langle l \otimes l^{\prime}+(L \square L) \mid l, l^{\prime} \in L\right\rangle=\left\langle l \wedge l^{\prime} \mid l, l^{\prime} \in L\right\rangle,
$$

which is called exterior product of $L$. From $[4,11,12]$, we may deduce that the following diagram (with Lie algebras and Lie homomorphisms) is commutative and has exact rows and central extensions as columns:

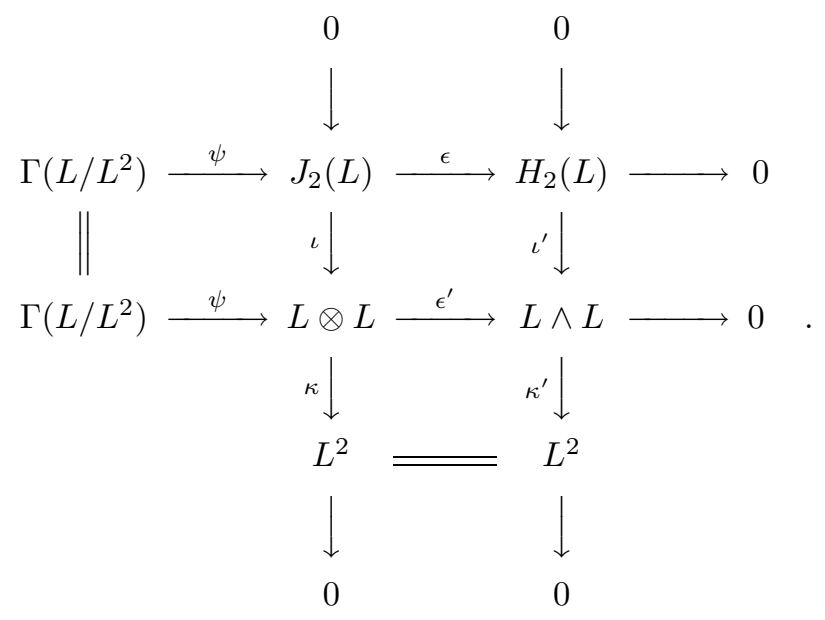

Here $\kappa$ is defined by $l \otimes l^{\prime} \in L \otimes L \mapsto\left[l, l^{\prime}\right] \in L^{2}, J_{2}(L)=\operatorname{ker} \kappa$ and contains $L \square L$, $\iota$ is the embedding of $J_{2}(L)$ into $L \otimes L, \epsilon$ is the natural homomorphism of $J_{2}(L)$ onto $H_{2}(L), \kappa^{\prime}$ is defined by $l \wedge l^{\prime} \in L \wedge L \mapsto\left[l, l^{\prime}\right] \in L^{2}, H_{2}(L)=\operatorname{ker} \kappa^{\prime}$ is the second homology Lie algebra of $L, \iota^{\prime}$ is the embedding of $H_{2}(L)$ into $L \wedge L, \epsilon^{\prime}$ is the natural homomorphism of $L \otimes L$ onto $L \wedge L, \Gamma$ is the Whitehead's universal quadratic functor in [4] and $\psi$ the Whitehead's universal quadratic homomorphism in [4]. It is interesting to note that there exists a Lie isomorphism in the sense of $[4$, Theorem 36 ] among the Lie algebras $H_{2}(L)$ and $M(L)$ (see also [11, Section 1]). 
Some recent contributions on the structure of $L \square L$ have been shown in $[11,12]$ and are recalled below.

LEMMA 4.1 (See [11], Lemma 2.1). Let $L$ be a nilpotent $n$-dimensional Lie algebra. Then $L \square L \simeq L / L^{2} \square L / L^{2}$.

This allows us to conclude the next result.

THEOREM 4.2. An $n$-dimensional nilpotent Lie algebra $L$ with $\operatorname{dim}\left(L / L^{2}\right)=d$ has $\operatorname{dim}(L \otimes L) \leq n d$ and the equality holds if and only if $L \simeq H(1)$ or $L \simeq A(n)$.

Proof. From the diagram above, we deduce that

$$
\operatorname{dim}(L \otimes L)=\operatorname{dim}(M(L))+\operatorname{dim}(L \square L)+\operatorname{dim}\left(L^{2}\right)
$$

and from Theorem 3.5 and Lemma 4.1 we may continue as follows

$$
\leq \frac{d-d^{2}+2 d n-2 n}{2}+\frac{d(d+1)}{2}+(n-d)=n d
$$

In the case that $L$ is abelian, the result holds. Otherwise, $L$ is non-abelian and the result follows by Lemma 3.2 and Theorem 3.1.

REMARK 4.3. We note that the inequality in Theorem 4.2 can be found in [14] in the case of groups and has been recently adapted to Lie algebras in [16, Corollary 3.4]. Here, we are using a different argument in the proof and are able to characterize all those nilpotent Lie algebras which achieve the bound.

We conclude with an improvement of Theorem 4.2.

Corollary 4.4. An $n$-dimensional nilpotent Lie algebra $L$ with $\operatorname{dim}\left(L / L^{2}\right)=d$, $\operatorname{dim}(Z(L))=t$ and $\operatorname{dim}\left(Z(L) \cap L^{2}\right)=s$ has

$$
d^{2} \leq \operatorname{dim}(L \otimes L) \leq n d-(n-d)(t-s)
$$

Proof. The upper bound follows by looking at the proof of Theorem 4.2.

The lower bound is obtained from $d^{2}=\operatorname{dim}\left(L / L^{2} \otimes_{\mathbb{Z}} L / L^{2}\right) \leq \operatorname{dim}(L \otimes L)$.

Acknowledgment. We are grateful to the referee for the careful reading. The second author thanks also the University of Molise for the support. The second author thanks also the University of Molise and the University of Palermo for the support. 


\section{REFERENCES}

[1] P. Batten, K. Moneyhun, and E. Stitzinger. On characterizing nilpotent Lie algebras by their multipliers. Communications in Algebra, 24:4319-4330, 1996.

[2] P. Batten and E. Stitzinger. On covers of Lie algebras. Communications in Algebra, 24:43014317, 1996.

[3] L. Bosko. On Schur Multipliers of Lie algebras and groups of maximal class. International Journal of Algebra and Computation, 20:807-821, 2010.

[4] G. Ellis. A non-abelian tensor product of Lie algebras. Glasgow Mathematical Journal, 39:101$120,1991$.

[5] P. Hardy and E. Stitzinger. On characterizing nilpotent Lie algebras by their multipliers $t(L)=3,4,5,6$. Communications in Algebra, 26:3527-3539, 1998.

[6] G. Karpilovsky. The Schur Multiplier. The Clarendon Press, Oxford University Press, New York, 1987.

[7] E. Khukhro. Nilpotent Groups and Their Autormoprhisms. Walter de Gruyter \& Co., Berlin, 1993.

[8] K. Moneyhun. Isoclinisms in Lie algebras. Algebras, Groups and Geometries, 11:9-22, 1994.

[9] P. Niroomand. On the order of Schur multiplier of non-abelian $p$-groups. Journal of Algebra, 322:4479-4482, 2009.

[10] P. Niroomand. The Schur multiplier of $p$-groups with large derived subgroup. Archiv der Mathematik (Basel), 95:101-103, 2010.

[11] P. Niroomand. Some properties on the tensor square of Lie algebras. Cornell University Library, E-print, arXiv:1006.2240, 2010.

[12] P. Niroomand. On the tensor square of non-abelian nilpotent finite dimensional Lie algebras. Linear and Multilinear Algebra, to appear.

[13] P. Niroomand and F.G. Russo. A note on the Schur multiplier of a nilpotent Lie algebra. Communications in Algebra, to appear.

[14] N.R. Rocco. On a construction related to the non-abelian tensor square of a group. Boletim da Sociedade Brasileira de Matemática, 22:63-79, 1991.

[15] J. Rotman. An Introduction to Homological Algebra. Academic Press, Inc., San Diego, 1979.

[16] A. Salemkar, H. Tavallaee, H. Mohammadzadeh, and B. Edalatzadeh. On the non-abelian tensor product of Lie algebras. Linear and Multilinear Algebra, 58:333-341, 2010.

[17] I. Schur. Untersuchungen über die Darstellung der endlichen Gruppen durch gebrochene lineare Substitutionen. Journal für die Reine und Angewandte Mathematik, 132: 85-137, 1907.

[18] B. Yankosky. On the multiplier of a Lie algebra. Journal of Lie Theory, 13:1-6, 2003.

[19] X.M. Zhou. On the order of the Schur multiplier of finite $p$-groups. Communications in Algebra, 22:1-8, 1994. 